\title{
Solvability and algorithms of generalized nonlinear variational-like inequalities in reflexive Banach spaces
}

\author{
Haiyan Gao ${ }^{1,2}$, Lili Wang ${ }^{3}$ and Liangshi Zhao ${ }^{4^{*}}$ (D
}

\begin{tabular}{l}
\hline${ }^{*}$ Correspondence: \\
liangshizhao@Innu.edu.cn \\
${ }^{4}$ Center for Studies of Marine \\
Economy and Sustainable \\
Development, Liaoning Normal \\
University, Dalian, Liaoning 116029, \\
People's Republic of China \\
Full list of author information is \\
available at the end of the article
\end{tabular}

available at the end of the article

\begin{abstract}
This paper deals with solvability and algorithms for a new class of generalized nonlinear variational-like inequalities in reflexive Banach spaces. By employing the Banach's fixed point theorem, Schauder's fixed point theorem, and FanKKM theorem, we obtain a sufficient condition which guarantees the existence of solutions for the generalized nonlinear variational-like inequality. We introduce also an auxiliary variational-like inequality and, by utilizing the minimax inequality, get the existence and uniqueness of solutions for the auxiliary variational-like inequality, which is used to suggest an iterative algorithm for solving the generalized nonlinear variational-like inequality. Under certain conditions, by means of the auxiliary principle technique, we both establish the existence and uniqueness of solutions for the generalized nonlinear variational-like inequality and discuss the convergence of iterative sequences generated by the iterative algorithm. Our results extend, improve, and unify several known results in the literature.
\end{abstract}

MSC: $47 J 20$

Keywords: Generalized nonlinear variational-like inequality; Banach's fixed point theorem; Schauder's fixed point theorem; FanKKM theorem; Iterative algorithm; Auxiliary principle technique; Minimax inequality; Reflexive Banach space

\section{Introduction}

Variational inequality is a powerful tool for studying problems arising in optimization, economics, differential equations, engineering and structural analysis, etc. For details, we refer to $[3,5,16]$ and the references therein. In 1988, Cohen [6] extended an auxiliary principle technique to study the existence of solutions for a class of variational inequalities. In 1994, Yao [16] obtained the existence of solutions for generalized variational inequalities in Banach spaces. Later, Chang-Xiang [5] investigated the existence of solutions for a class of quasibilinear variational inequalities by making use of the minimax inequality due to themselves in Hilbert spaces. In 2012, Yao-Postolache [17] introduced an iterative scheme for finding a common element of the set of solution of a pseudomonotone, Lipschitz-continuous variational inequality problem and the set of common fixed points of an infinite family of nonexpansive mappings, and showed a few necessary and sufficient

(c) The Author(s) 2020. This article is licensed under a Creative Commons Attribution 4.0 International License, which permits use sharing, adaptation, distribution and reproduction in any medium or format, as long as you give appropriate credit to the original author(s) and the source, provide a link to the Creative Commons licence, and indicate if changes were made. The images or other third party material in this article are included in the article's Creative Commons licence, unless indicated otherwise in a credit line to the material. If material is not included in the article's Creative Commons licence and your intended use is not permitted by statutory regulation or exceeds the permitted use, you will need to obtain permission directly from the copyright holder. To view a copy of this licence, visit http://creativecommons.org/licenses/by/4.0/. 
conditions for strong convergence of the sequences generated by the proposed scheme. In 2016, Yao-Postolache-Liou-Yao [19] introduced a monotone variational inequality in Hilbert spaces, suggested an implicit algorithm, and proved its convergence hierarchical to the solution of the monotone variational inequality. Recently, Yao-Postolache-Yao [23] considered the fixed point and variational inequality problems in Hilbert spaces, suggested an extragradient algorithm, and proved strong convergence of the proposed algorithm, while Yao-Postolache-Yao [21] introduced a generalized variational inequality in Hilbert spaces, constructed an iterative algorithm for solving the generalized variational inequality, and obtained strong convergence of the algorithm.

Variational-like inequality and generalized variational-like inequality, known as useful and important generalized forms of variational inequalities, were also discussed and analyzed by many authors. For details, we refer to $[1,7,8,11,13,24,25]$ and the references therein. Especially, Liu-Ume-Kang [13] and Zeng [24] established some existence and uniqueness theorems of solutions for generalized nonlinear variational-like inequalities in reflexive Banach spaces by applying the minimax inequality due to Ding-Tan [9].

Stimulated and inspired by the recent results in [1-26], we introduce a new generalized nonlinear variational-like inequality which includes these variational inequalities and variational-like inequalities in $[6-8,16,24]$ as special cases. Next, the Banach's fixed point theorem, Schauder's fixed point theorem, and FanKKM theorem are applied to prove the existence of a solution for the generalized nonlinear variational-like inequality. Moreover, in order to suggest an iterative algorithm for computing the approximate solutions of the generalized nonlinear variational-like inequality, an auxiliary variational-like inequality is introduced and the existence and uniqueness of the solution for the auxiliary variationallike inequality is proved by using the minimax inequality due to Ding-Tan [9]. Finally, both the existence and uniqueness of solutions for the generalized nonlinear variationallike inequality and the convergence of iterative sequences presented in the algorithm are discussed under certain conditions.

\section{Preliminaries}

Throughout this paper, unless other specified, we always assume that $\mathbb{R}=(-\infty,+\infty)$, $\mathbb{N}$ and $\omega$ stand for the sets of all positive and nonnegative integers, respectively, $D$ is a nonempty bounded closed convex subset of a reflexive Banach space $B$ with the dual space $B^{*}$ and $\langle u, v\rangle$ is the dual pairing between $u \in B^{*}$ and $v \in B$. Assume that the functional $b: D \times D \rightarrow \mathbb{R}$ satisfies the following conditions:

(b1) $b$ is linear in the first argument and convex in the second argument;

(b2) there exists a constant $\gamma>0$ satisfying $b(x, y) \leq \gamma\|x\|\|y\|, \forall x, y \in D$;

(b3) $b(x, y)-b(x, z) \leq b(x, y-z), \forall x, y, z \in D$.

It follows that

$b$ is continuous in the second argument on $D$.

Let $E, F: D \rightarrow B^{*}, N: B^{*} \times B^{*} \rightarrow B^{*}, \eta: D \times D \rightarrow B$, and $g: D \rightarrow D$ be five mappings. We shall investigate the following generalized nonlinear variational-like inequality: determine $u \in D$ such that

$$
\langle N(E u, F u), \eta(v, u)\rangle+b(g u, v)-b(g u, u) \geq 0, \quad \forall v \in D,
$$

where $b$ satisfies (b1)-(b3) and $b$ is not necessarily differentiable. 


\section{Special Cases:}

(A) If $N(x, y)=x-y, \forall x, y \in B^{*}$ and $g=I$ (the identity mapping in $D$ ), then problem (2.2) collapses to finding $u \in D$ such that

$$
\langle E u-F u, \eta(v, u)\rangle+b(u, v)-b(u, u) \geq 0, \quad \forall v \in D
$$

which was introduced and studied by Ding [8].

(B) If $b(u, v)=f(v), \forall u, v \in D$, where $f: D \rightarrow \mathbb{R}$ is a mapping, then problem (2.3) reduces to seeking $u \in D$ such that

$$
\langle E u-F u, \eta(v, u)\rangle+f(v)-f(u) \geq 0, \quad \forall v \in D,
$$

which is known as a mixed nonlinear variational-like inequality and was discussed by Ding [7] and Zeng [24] in Banach and Hilbert spaces, respectively.

(C) If $\eta(v, u)=g u-g v, \forall u, v \in D$, where $g: D \rightarrow B$ is a mapping, then problem (2.4) is equivalent to finding $u \in D$ such that

$$
\langle E u-F u, g u-g v\rangle+f(v)-f(u) \geq 0, \quad \forall v \in D .
$$

Yao [16] investigated problem (2.5) which included the variational inequalities introduced by Cohen [6] as a special case.

In brief, there are a number of special cases of problems (2.2)-(2.5) for suitable choices of the mappings $N, E, F, \eta, g$, and the functional $b$, which can be found in $[6-8,16,24]$ and the references cited therein.

We need the following definitions and results which will be used in the paper.

Definition 2.1 Let $g: D \rightarrow D, F: D \rightarrow B^{*}, \eta: D \times D \rightarrow B$, and $N: B^{*} \times B^{*} \rightarrow B^{*}$ be mappings.

(1) $N$ is said to be Lipschitz continuous in the first argument if there exists a constant $\alpha>0$ such that

$$
\|N(u, x)-N(v, x)\| \leq \alpha\|u-v\|, \quad \forall u, v \in D, x \in B^{*} ;
$$

(2) $N$ is said to be $\eta$-strongly monotone with respect to $F$ in the second argument if there exists a constant $\beta>0$ such that

$$
\langle N(x, F u)-N(x, F v), \eta(u, v)\rangle \geq \beta\|u-v\|^{2}, \quad \forall u, v \in D, x \in B^{*} ;
$$

(3) $\eta$ is said to be Lipschitz continuous if there exists a constant $\delta>0$ such that

$$
\|\eta(u, v)\| \leq \delta\|u-v\|, \quad \forall u, v \in D
$$

(4) $g$ is said to be Lipschitz continuous if there exists a constant $r>0$ such that

$$
\|g u-g v\| \leq r\|u-v\|, \quad \forall u, v \in D .
$$


Definition 2.2 Let $E, F: D \rightarrow B^{*}, N: B^{*} \times B^{*} \rightarrow B^{*}$, and $\eta: D \times D \rightarrow B$ be mappings. For any $u_{1} \in D$, the mappings $F, N\left(E u_{1}, \cdot\right)$, and $\eta$ are said to have 0 -diagonally concave relation on $D$ if the function $\psi: D \times D \rightarrow \mathbb{R} \cup\{+\infty\}$ defined by

$$
\psi(u, v)=\left\langle N\left(E u_{1}, F u\right), \eta(u, v)\right\rangle, \quad \forall u, v \in D,
$$

is 0-diagonally concave on $D$, that is, for any finite set $\left\{v_{1}, \ldots, v_{m}\right\} \subset D$ and for any $u=$ $\sum_{i=1}^{m} \lambda_{i} v_{i}$ with $\lambda_{i} \geq 0$ and $\sum_{i=1}^{m} \lambda_{i}=1$,

$$
\sum_{i=1}^{m} \lambda_{i} \psi\left(u, v_{i}\right) \leq 0
$$

Definition 2.3 Let $X$ and $Y$ be topological spaces. A mapping $f: X \rightarrow Y$ is said to be compact if it is continuous and has a relatively compact range.

Definition 2.4 ([1]) Let $D$ be a nonempty convex subset of a Banach space $B$ and $h: D \rightarrow$ $\mathbb{R}$ be a Fréchet differentiable function. Then $h$ is said to be

(1) $\eta$-convex if

$$
h(v)-h(u) \geq\left\langle h^{\prime}(u), \eta(v, u)\right\rangle, \quad \forall u, v \in D
$$

(2) $\eta$-strongly convex if there exists a constant $\mu>0$ satisfying

$$
h(v)-h(u)-\left\langle h^{\prime}(u), \eta(v, u)\right\rangle \geq \frac{\mu}{2}\|u-v\|^{2}, \quad \forall u, v \in D .
$$

Lemma 2.1 (Schauder's Fixed Point Theorem) Let D be a nonempty convex subset of a normed linear space $X$ and $f: D \rightarrow D$ be compact. Then $f$ has a fixed point in $D$.

For $D \subseteq B$, we define by $\operatorname{conv}(D)$ the convex hull of $B$. The set-valued mapping $P: D \rightarrow$ $2^{D}$ is said to be a KKM mapping, if for any finite subset $\left\{v_{1}, \ldots, v_{m}\right\}$ of $D$,

$$
\operatorname{conv}\left(\left\{v_{1}, \ldots, v_{m}\right\}\right) \subseteq \bigcup_{i=1}^{m} P\left(v_{i}\right)
$$

Lemma 2.2 (FanKKM Theorem, [10]) Let D be an arbitrary nonempty set in a topological vector space $B$, and let $P: D \rightarrow 2^{D}$ be a KKM mapping. If $P(v)$ is closed for each $v \in D$ and is compact for at least one $v \in B$, then $\bigcap_{v \in D} P(v) \neq \emptyset$.

Lemma 2.3 ([1]) Let $\eta: D \times D \rightarrow B$ be continuous from the weak topology to the weak topology. Let $h: D \rightarrow \mathbb{R}$ be Fréchet differentiable such that $h^{\prime}$ is continuous from the weak topology to the strong topology. Then the mapping $g: D \rightarrow \mathbb{R}$ defined by $g(x)=\left\langle h^{\prime}(x), \eta(y, x)\right\rangle$ for each fixed $y \in D$ is also continuous from the weak topology to the strong topology.

Lemma 2.4 ([9]) Let D be a nonempty convex subset of a topological vector space and let $\varphi: D \times D \rightarrow \mathbb{R} \cup\{+\infty\}$ be such that

(a) for each $v \in D, \varphi(v, \cdot)$ is lower semicontinuous on each nonempty compact subset of $D$; 
(b) for each nonempty finite set $\left\{v_{1}, \ldots, v_{m}\right\} \subset D$ and for any $u=\sum_{i=1}^{m} \lambda_{i} v_{i}$ with $\lambda_{i} \geq 0$ and $\sum_{i=1}^{m} \lambda_{i}=1, \min _{1 \leq i \leq m} \varphi\left(v_{i}, u\right) \leq 0$;

(c) there exist a nonempty compact convex subset $X_{0}$ of $D$ and a nonempty compact subset $K$ of $D$ such that for each $v \in D \backslash K$ there is $u \in \operatorname{co}\left(X_{0} \cup\{v\}\right)$ with $\varphi(u, v)>0$.

Then there exists $\hat{v} \in K$ such that $\varphi(u, \hat{v}) \leq 0$ for all $u \in D$.

\section{Existence of solutions for the generalized nonlinear variational-like inequality (2.2)}

This section is devoted to the existence result of solutions for the generalized nonlinear variational-like inequality (2.2) by employing the Banach's fixed point theorem, Schauder's fixed point theorem, and FanKKM theorem.

Theorem 3.1 Let $E: D \rightarrow B^{*}$ be a compact mapping, $F: D \rightarrow B^{*}, N: B^{*} \times B^{*} \rightarrow B^{*}, \eta:$ $D \times D \rightarrow B, g: D \rightarrow D$ be four mappings, and $b: D \times D \rightarrow \mathbb{R} \cup\{ \pm \infty\}$ be a functional satisfying (b1)-(b3). Assume that

(c1) $F$ is continuous, for each $u_{1} \in D, N\left(E u_{1}, \cdot\right)$ is continuous on $D$, and the mappings $F$, $N\left(E u_{1}, \cdot\right)$, and $\eta$ have the 0 -diagonally concave relation on $D$;

(c2) $\eta$ is Lipschitz continuous with a constant $\delta>0$ and for any $v \in D, \eta(\cdot, v)$ is continuous from the weak topology to the weak topology;

(c3) $N$ is Lipschitz continuous and $\eta$-strongly monotone with respect to $F$ in the first and second argument with constants $\alpha>0, \beta>0$, respectively, and $g$ is Lipschitz continuous with a constant $r>0$;

(c4) $\eta(u, v)=-\eta(v, u), \forall u, v \in D$, and $\beta>\gamma r$.

Then the generalized nonlinear variational-like inequality (2.2) has a solution in $D$.

Proof First of all, let $u_{1}$ be an arbitrary fixed element in $D$. For each $u_{0} \in D$, we show that there exists a unique $\hat{w} \in D$ such that

$$
\left\langle N\left(E u_{1}, F \hat{w}\right), \eta(v, \hat{w})\right\rangle+b\left(g u_{0}, v\right)-b\left(g u_{0}, \hat{w}\right) \geq 0, \quad \forall v \in D .
$$

Define a set-valued mapping $P: D \rightarrow 2^{D}$ by

$$
P(v)=\left\{\hat{w} \in D:\left\langle N\left(E u_{1}, F \hat{w}\right), \eta(v, \hat{w})\right\rangle+b\left(g u_{0}, v\right)-b\left(g u_{0}, \hat{w}\right) \geq 0\right\}, \quad \forall v \in D .
$$

Obviously, $v \in P(v) \neq \emptyset, \forall v \in D$. Next we claim that $P$ is a KKM mapping. Otherwise, there exists a finite set $\left\{v_{1}, v_{2}, \ldots, v_{m}\right\} \subset D$ and $\lambda_{i} \geq 0, i \in\{1,2, \ldots, m\}$ with $\sum_{i=1}^{m} \lambda_{i}=1$ such that

$$
w=\sum_{i=1}^{m} \lambda_{i} v_{i} \bar{\in} P\left(v_{i}\right), \quad \forall i \in\{1,2, \ldots, m\}
$$

That is,

$$
\left\langle N\left(E u_{1}, F w\right), \eta\left(v_{i}, w\right)\right\rangle+b\left(g u_{0}, v_{i}\right)-b\left(g u_{0}, w\right)<0, \quad \forall i \in\{1,2, \ldots, m\}
$$

which implies that by (b1), (c1), and (c4)

$$
0<\sum_{i=1}^{m} \lambda_{i} b\left(g u_{0}, v_{i}\right)-b\left(g u_{0}, w\right)<\sum_{i=1}^{m} \lambda_{i}\left\langle N\left(E u_{1}, F w\right), \eta\left(w, v_{i}\right)\right\rangle \leq 0,
$$


which is a contradiction. Hence $P: D \rightarrow 2^{D}$ is a KKM mapping. Since $\overline{P(v)}^{w}$ is a weakly closed subset of the bounded set $D$, it is weakly compact. It follows from Lemma 2.2 that $\bigcap_{v \in D} \overline{P(v)}^{w} \neq \emptyset$. Let $\hat{w} \in \bigcap_{v \in D} \overline{P(v)}^{w}$. Thus there is a sequence $\left\{w_{m}\right\}_{m \in \mathbb{N}} \subseteq P(v), \forall v \in D$, such that $w_{m} \rightarrow \hat{w} \in D$ as $m \rightarrow \infty$. It follows that

$$
\left\langle N\left(E u_{1}, F w_{m}\right), \eta\left(v, w_{m}\right)\right\rangle+b\left(g u_{0}, v\right)-b\left(g u_{0}, w_{m}\right) \geq 0, \quad \forall v \in D, m \in \mathbb{N},
$$

and further

$$
\lim _{m \rightarrow \infty}\left\langle N\left(E u_{1}, F w_{m}\right), \eta\left(v, w_{m}\right)\right\rangle+b\left(g u_{0}, v\right)-\lim _{m \rightarrow \infty} b\left(g u_{0}, w_{m}\right) \geq 0, \quad \forall v \in D
$$

By (c1), (c2), and (2.1), we gain that (3.1) holds. Namely, (3.1) possesses a solution $\hat{w} \in D$ for any $u_{0} \in D$. Now we prove the uniqueness of solution for (3.1) with respect to $u_{0} \in D$. Suppose that $\bar{w} \in D \backslash\{\hat{w}\}$ is also a solution of (3.1) with respect to $u_{0} \in D$. It follows that

$$
\left\langle N\left(E u_{1}, F \bar{w}\right), \eta(v, \bar{w})\right\rangle+b\left(g u_{0}, v\right)-b\left(g u_{0}, \bar{w}\right) \geq 0, \quad \forall v \in D
$$

Taking $v=\bar{w}$ in (3.1), $v=\hat{w}$ in (3.2) and adding them together, we get that in view of (c3),

$$
\beta\|\hat{w}-\bar{w}\|^{2} \leq\left\langle N\left(E u_{1}, F \hat{w}\right)-N\left(E u_{1}, F \bar{w}\right), \eta(\hat{w}, \bar{w})\right\rangle \leq 0,
$$

which implies that $\beta \leq 0$, a contradiction. That is, $\hat{w}$ is the unique solution of (3.1) with respect to $u_{0} \in D$. It follows that there exists a mapping $f: D \rightarrow D$ such that for each $u_{0} \in D, f u_{0}$ is the unique solution of (3.1).

Secondly, for each $u_{1} \in D$, we show that there exists a unique $w_{0} \in D$ satisfying

$$
\left\langle N\left(E u_{1}, F w_{0}\right), \eta\left(v, w_{0}\right)\right\rangle+b\left(g w_{0}, v\right)-b\left(g w_{0}, w_{0}\right) \geq 0, \quad \forall v \in D
$$

In fact, for every $x, y \in D$, there exist $w_{1}=f(x), w_{2}=f(y)$ such that

$$
\begin{aligned}
& \left\langle N\left(E u_{1}, F w_{1}\right), \eta\left(v, w_{1}\right)\right\rangle+b(g x, v)-b\left(g x, w_{1}\right) \geq 0, \\
& \left\langle N\left(E u_{1}, F w_{2}\right), \eta\left(v, w_{2}\right)\right\rangle+b(g y, v)-b\left(g y, w_{2}\right) \geq 0,
\end{aligned}
$$

for each $v \in D$. Taking $v=w_{2}$ in (3.4), $v=w_{1}$ in (3.5) and adding these two inequalities, we know that by (b2), (b3), (c3), and (c4),

$$
\begin{aligned}
\beta\left\|w_{1}-w_{2}\right\|^{2} & \leq\left\langle N\left(E u_{1}, F w_{1}\right)-N\left(E u_{1}, F w_{2}\right), \eta\left(w_{1}, w_{2}\right)\right\rangle \\
& \leq b\left(g x-g y, w_{2}\right)-b\left(g x-g y, w_{1}\right) \\
& \leq \gamma\|g x-g y\|\left\|w_{1}-w_{2}\right\| \\
& \leq \gamma r\|x-y\|\left\|w_{1}-w_{2}\right\|,
\end{aligned}
$$

which means that

$$
\left\|w_{1}-w_{2}\right\| \leq \frac{\gamma r}{\beta}\|x-y\|, \quad \forall x, y \in D .
$$


It follows from (c4) that $f$ is a contraction mapping on $D$ and so it has a unique fixed point $w_{0} \in D$ satisfying (3.3) according to the Banach's fixed point theorem. We now verify that $w_{0}$ is the unique solution of (3.3) relative to $u_{1} \in D$. Suppose that $w_{0}^{\prime} \in D \backslash\left\{w_{0}\right\}$ is another solution of (3.3) relative to $u_{1} \in D$, that is,

$$
\left\langle N\left(E u_{1}, F w_{0}^{\prime}\right), \eta\left(v, w_{0}^{\prime}\right)\right\rangle+b\left(g w_{0}^{\prime}, v\right)-b\left(g w_{0}^{\prime}, w_{0}^{\prime}\right) \geq 0, \quad \forall v \in D .
$$

Take $v=w_{0}^{\prime}$ in (3.3), $v=w_{0}$ in (3.6) and add (3.3) and (3.6). Based on (b2), (b3), (c3), and (c4), we conclude that

$$
\begin{aligned}
\beta\left\|w_{0}-w_{0}^{\prime}\right\|^{2} & \leq\left\langle N\left(E u_{1}, F w_{0}\right)-N\left(E u_{1}, F w_{0}^{\prime}\right), \eta\left(w_{0}, w_{0}^{\prime}\right)\right\rangle \\
& \leq b\left(g w_{0}-g w_{0}^{\prime}, w_{0}^{\prime}\right)-b\left(g w_{0}-g w_{0}^{\prime}, w_{0}\right) \\
& \leq \gamma r\left\|w_{0}-w_{0}^{\prime}\right\|^{2}
\end{aligned}
$$

which contradicts (c4). Therefore, $w_{0}$ is the unique solution of (3.3) relative to $u_{1} \in D$. Hence for each $u_{1} \in D$, there exists a mapping $h: D \rightarrow D$ such that $h u_{1}$ is the unique solution of (3.3).

Finally, we show that $h$ is a compact mapping on $D$. By the definition of $h$, we obtain that

$$
\begin{aligned}
& \langle N(E x, F h x), \eta(v, h x)\rangle+b(g h x, v)-b(g h x, h x) \geq 0, \\
& \langle N(E y, F h y), \eta(v, h y)\rangle+b(g h y, v)-b(g h y, h y) \geq 0,
\end{aligned}
$$

for every $x, y, v \in D$. Letting $v=h y$ in (3.7), $v=h x$ in (3.8) and adding them together, by employing (b1)-(b3) and (c2)-(c4), we arrive at

$$
\begin{aligned}
0 \leq & b(g h x-g h y, h y)-b(g h x-g h y, h x)-\langle N(E x, F h x)-N(E y, F h x), \eta(h x, h y)\rangle \\
& -\langle N(E y, F h x)-N(E y, F h y), \eta(h x, h y)\rangle \\
\leq & \gamma\|g h x-g h y\|\|h x-h y\|+\|N(E x, F h x)-N(E y, F h x)\|\|\eta(h x, h y)\| \\
& -\beta\|h x-h y\|^{2} \\
\leq & (\gamma r-\beta)\|h x-h y\|^{2}+\alpha \delta\|E x-E y\|\|h x-h y\|,
\end{aligned}
$$

which reduces to

$$
\|h x-h y\| \leq \frac{\alpha \delta}{\beta-\gamma r}\|E x-E y\|, \quad \forall x, y \in D .
$$

Since $E$ is a compact mapping, it is easy to verify that $h$ is also compact. The Schauder's fixed point theorem yields that there exists some $u \in D$ such that $h u=u$. Consequently, $u \in D$ is a solution of problem (2.2). This completes the proof.

Remark 3.1 Theorem 3.1 extends and improves the corresponding results in [1, 6$8,16,24]$. Not only these variational and variational-like inequalities in $[6-8,16,24]$ are replaced by the more generalized nonlinear variational-like inequality (2.2), but also Theorem 3.1 first combines the Banach's fixed point theorem, Schauder's fixed point theorem, and FanKKM theorem to establish the existence of solutions for the generalized nonlinear variational-like inequality (2.2). 


\section{Existence and uniqueness of solutions for the auxiliary variational-like inequality and iterative algorithm}

In this section, we introduce an auxiliary variational-like inequality and establish an existence and uniqueness theorem of the solution for the auxiliary variational-like inequality by applying the minimax inequality due to Ding-Tan [9]. Based on this theorem, we suggest a new iterative algorithm to compute the approximate solutions of the generalized nonlinear variational-like inequality (2.2).

Let $K: D \rightarrow \mathbb{R} \cup\{+\infty\}$ be a given Fréchet differentiable $\eta$-strongly convex functional and $\rho>0$ be a constant. Let $\theta$ be a constant in $[0,1]$ and $x$ be any fixed element in $D$. For each $u \in D$, we consider the following auxiliary variational-like inequality: Find $w \in D$ such that

$$
\begin{aligned}
& (1-\theta)\left\langle K^{\prime}(w)-K^{\prime}(u), \eta(v, w)\right\rangle \\
& \quad \geq \theta \rho\langle N(E x, F w), \eta(w, v)\rangle+\theta \rho b(g u, w)-\theta \rho b(g u, v), \quad \forall v \in D .
\end{aligned}
$$

Theorem 4.1 Let $E, F: D \rightarrow B^{*}, N: B^{*} \times B^{*} \rightarrow B^{*}, \eta: D \times D \rightarrow B, g: D \rightarrow D$ be five mappings and $b: D \times D \rightarrow \mathbb{R} \cup\{ \pm \infty\}$ be a functional satisfying (b1)-(b3). Let $K: D \rightarrow$ $\mathbb{R} \cup\{+\infty\}$ be a differentiable functional. If $(c 1),(c 2)$ and the following conditions hold:

(d1) $N$ is $\eta$-strongly monotone with respect to $F$ in the second argument with a constant $\beta>0$;

(d2) $\eta(u, v)=\eta(u, w)+\eta(w, v), \forall u, v, w \in D$;

(d3) $K$ is $\eta$-strongly convex with a constant $\mu>0$ and $K^{\prime}$ is sequentially continuous from the weak topology to the strongly topology,

then the auxiliary variational-like inequality (4.1) processes a solution in D with respect to $u \in D$.

Proof Let $x$ be any fixed element in $D$. For each $u \in D$, define a functional $\varphi: D \times D \rightarrow$ $\mathbb{R} \cup\{ \pm \infty\}$ by

$$
\begin{aligned}
\varphi(v, w)= & (1-\theta)\left\langle K^{\prime}(u)-K^{\prime}(w), \eta(v, w)\right\rangle+\theta \rho\langle N(E x, F w), \eta(w, v)\rangle \\
& +\theta \rho b(g u, w)-\theta \rho b(g u, v), \quad \forall v, w \in D .
\end{aligned}
$$

It is not difficult to verify that condition (a) of Lemma 2.4 is satisfied. Next, we claim that $\varphi(v, w)$ satisfies condition (b) of Lemma 2.4. If it were false, there would exist a finite set $\left\{v_{1}, \ldots, v_{m}\right\} \subset D$ and $w=\sum_{i=1}^{m} \lambda_{i} v_{i}$ with $\lambda_{i} \geq 0$ and $\sum_{i=1}^{m} \lambda_{i}=1$ such that $\varphi\left(v_{i}, w\right)>0$ for any $i \in\{1,2, \ldots, m\}$. It follows from this that

$$
\begin{aligned}
0< & (1-\theta) \sum_{i=1}^{m} \lambda_{i}\left\langle K^{\prime}(u)-K^{\prime}(w), \eta\left(v_{i}, w\right)\right\rangle \\
& +\theta \rho \sum_{i=1}^{m} \lambda_{i}\left\langle N(E x, F w), \eta\left(w, v_{i}\right)\right\rangle+\theta \rho b(g u, w)-\theta \rho \sum_{i=1}^{m} \lambda_{i} b\left(g u, v_{i}\right) \\
= & \theta \rho \sum_{i=1}^{m} \lambda_{i}\left\langle N(E x, F w), \eta\left(w, v_{i}\right)\right\rangle+\theta \rho b(g u, w)-\theta \rho \sum_{i=1}^{m} \lambda_{i} b\left(g u, v_{i}\right) \leq 0
\end{aligned}
$$

which is impossible. Hence condition (b) of Lemma 2.4 is fulfilled. For a given $v^{*} \in D$, put $X=\left\{v^{*}\right\}$ and $Y=\left\{w \in D:\left\|v^{*}-w\right\| \leq R\right\}$, where $R=\frac{1}{(1-\theta) \mu+\theta \rho \beta}\left[(1-\theta) \delta\left\|K^{\prime}(u)-K^{\prime}\left(v^{*}\right)\right\|+\right.$ 
$\left.\theta \rho \delta\left\|N\left(E x, F v^{*}\right)\right\|+\theta \rho \gamma\|g u\|\right]$. It is clear that $X$ and $Y$ are both weakly compact convex subsets of $D$. By virtue of (b2), (b3), (c2), (d1), and (d3), we gain that for each $w \in D \backslash Y$, there is a $v^{*} \in \operatorname{co}(X \cup\{w\})$ such that

$$
\begin{aligned}
\psi\left(v^{*}, w\right)= & (1-\theta)\left\langle K^{\prime}(u)-K^{\prime}\left(v^{*}\right), \eta\left(v^{*}, w\right)\right\rangle+(1-\theta)\left\langle K^{\prime}\left(v^{*}\right)-K^{\prime}(w), \eta\left(v^{*}, w\right)\right\rangle \\
& +\theta \rho\left\langle N(E x, F w)-N\left(E x, F v^{*}\right), \eta\left(w, v^{*}\right)\right\rangle+\theta \rho\left\langle N\left(E x, F v^{*}\right), \eta\left(w, v^{*}\right)\right\rangle \\
& +\theta \rho b(g u, w)-\theta \rho b\left(g u, v^{*}\right) \\
\geq & -(1-\theta) \delta\left\|K^{\prime}(u)-K^{\prime}\left(v^{*}\right)\right\|\left\|v^{*}-w\right\|+(1-\theta) \mu\left\|v^{*}-w\right\|^{2} \\
& +\theta \rho \beta\left\|v^{*}-w\right\|^{2}-\theta \rho \delta\left\|N\left(E x, F v^{*}\right)\right\|\left\|v^{*}-w\right\|-\theta \rho \gamma\|g u\|\left\|v^{*}-w\right\| \\
= & \left\|v^{*}-w\right\|\left\{[(1-\theta) \mu+\theta \rho \beta]\left\|v^{*}-w\right\|-(1-\theta) \delta\left\|K^{\prime}(u)-K^{\prime}\left(v^{*}\right)\right\|\right. \\
& \left.-\theta \rho \delta\left\|N\left(E x, F v^{*}\right)\right\|-\theta \rho \gamma\|g u\|\right\} \\
> & 0 .
\end{aligned}
$$

Thus, condition (c) of Lemma 2.4 holds as well. As a result, Lemma 2.4 ensures that there exists some $w \in D$ such that $\varphi(v, w) \leq 0$ for all $v \in D$, namely, the auxiliary problem (4.1) has a solution $w \in D$ with respect to $u \in D$. Now we prove the uniqueness of the solution for the auxiliary problem (4.1) with respect to $u \in D$. Suppose that $\bar{w} \in D \backslash\{w\}$ is another solution of the auxiliary problem (4.1) relative to $u \in D$. It follows that

$$
\begin{aligned}
& (1-\theta)\left\langle K^{\prime}(\bar{w})-K^{\prime}(u), \eta(v, \bar{w})\right\rangle \\
& \quad \geq \theta \rho\langle N(E x, F \bar{w}), \eta(\bar{w}, v)\rangle+\theta \rho b(g u, \bar{w})-\theta \rho b(g u, v), \quad \forall v \in D .
\end{aligned}
$$

Taking $v=\bar{w}$ in (4.1), $v=w$ in (4.2) and adding them together, we obtain by (d1) $-(\mathrm{d} 3)$ that

$$
\begin{aligned}
0 \geq & -(1-\theta)\left\langle K^{\prime}(\bar{w}), \eta(w, \bar{w})\right\rangle-(1-\theta)\left\langle K^{\prime}(w), \eta(\bar{w}, w)\right\rangle \\
& +\theta \rho\langle N(E x, F \bar{w}), \eta(\bar{w}, w)\rangle+\theta \rho\langle N(E x, F w), \eta(w, \bar{w})\rangle \\
= & (1-\theta)\left\langle K^{\prime}(\bar{w})-K^{\prime}(w), \eta(\bar{w}, w)\right\rangle+\theta \rho\langle N(E x, F w)-N(E x, F \bar{w}), \eta(w, \bar{w})\rangle \\
\geq & {[(1-\theta) \mu+\theta \rho \beta]\|w-\bar{w}\|^{2}, }
\end{aligned}
$$

which implies that $(1-\theta) \mu+\theta \rho \beta \leq 0$, a contradiction. Therefore, $w$ is the unique solution of the auxiliary problem (4.1) relative to $u \in D$. This completes the proof.

Based on Theorem 4.1, we suggest the following iterative algorithm for the generalized nonlinear variational-like inequality (2.2).

Algorithm 4.1 For given $u_{0}, v_{0} \in D$, compute $\left\{u_{n}, v_{n}, w_{n}\right\}_{n \in \omega}$ by the following iterative scheme:

$$
\begin{aligned}
& \left(1-\alpha_{n}\right)\left\langle K^{\prime}\left(w_{n}\right)-K^{\prime}\left(u_{n}\right), \eta\left(v, w_{n}\right)\right\rangle \\
& \quad \geq \alpha_{n} \rho\left\langle N\left(E v_{n}, F w_{n}\right), \eta\left(w_{n}, v\right)\right\rangle+\alpha_{n} \rho b\left(g u_{n}, w_{n}\right)-\alpha_{n} \rho b\left(g u_{n}, v\right), \quad \forall v \in D, \\
& \left(1-\beta_{n}\right)\left\langle K^{\prime}\left(v_{n+1}\right)-K^{\prime}\left(w_{n}\right), \eta\left(v, v_{n+1}\right)\right\rangle \\
& \quad \geq \beta_{n} \rho\left\langle N\left(E u_{n}, F v_{n+1}\right), \eta\left(v_{n+1}, v\right)\right\rangle+\beta_{n} \rho b\left(g w_{n}, v_{n+1}\right)-\beta_{n} \rho b\left(g w_{n}, v\right), \quad \forall v \in D,
\end{aligned}
$$




$$
\begin{aligned}
& \left(1-\gamma_{n}\right)\left\langle K^{\prime}\left(u_{n+1}\right)-K^{\prime}\left(v_{n+1}\right), \eta\left(v, u_{n+1}\right)\right\rangle \\
& \geq \gamma_{n} \rho\left\langle N\left(E w_{n}, F u_{n+1}\right), \eta\left(u_{n+1}, v\right)\right\rangle \\
& \quad+\gamma_{n} \rho b\left(g v_{n+1}, u_{n+1}\right)-\gamma_{n} \rho b\left(g v_{n+1}, v\right), \quad \forall v \in D,
\end{aligned}
$$

where $\rho>0$ is a constant, $\left\{\alpha_{n}\right\}_{n \in \omega},\left\{\beta_{n}\right\}_{n \in \omega}$, and $\left\{\gamma_{n}\right\}_{n \in \omega}$ are arbitrary sequences in $[0,1]$ with

$$
\theta_{1}=\inf \left\{\alpha_{n}, \beta_{n}, \gamma_{n}: n \in \omega\right\}, \quad \theta_{2}=\sup \left\{\alpha_{n}, \beta_{n}, \gamma_{n}: n \in \omega\right\}
$$

Remark 4.1 Obviously, Algorithm 4.1 is much more novel than the corresponding algorithms of Ansari-Yao [1], Ding [7, 8], Liu-Chen-Kang-Ume [12], Liu-Ume-Kang [13], Yao [16], Zeng [24], and Zhang-Liu-Kang [25].

\section{Convergence of Algorithm 4.1}

Now we discuss the convergence of the iterative sequences $\left\{u_{n}\right\}_{n \in \omega},\left\{v_{n}\right\}_{n \in \omega}$, and $\left\{w_{n}\right\}_{n \in \omega}$ generated by Algorithm 4.1 depending on the existence of solutions for the generalized nonlinear variational-like inequality $(2.2)$.

Theorem 5.1 Let $E, F: D \rightarrow B^{*}, N: B^{*} \times B^{*} \rightarrow B^{*}, \eta: D \times D \rightarrow B, g: D \rightarrow D$ be mappings and $b: D \times D \rightarrow \mathbb{R} \cup\{ \pm \infty\}$ be a functional satisfying (b1)-(b3). Let $K: D \rightarrow \mathbb{R} \cup\{+\infty\}$ be a differentiable functional. Assume that (c1)-(c3), (d2), (d3) hold and

(e1) $N$ is Lipschitz continuous in the second argument with constants $s>0$, and $E$, $F$ are Lipschitz continuous with constants $l>0, t>0$, respectively.

Then (a) if

$$
\beta>\gamma r+\alpha \delta l,
$$

then the generalized nonlinear variational-like inequality (2.2) has a unique solution $u \in D$;

(b) for each $\rho>0, n \in \omega$ and $u_{0}, v_{0} \in D$, (4.3) has a unique solution $w_{n} \in D$ with respect to $u_{n} \in D$, (4.4) has a unique solution $v_{n+1} \in D$ with respect to $w_{n} \in D$, (4.5) has a unique solution $u_{n+1} \in D$ with respect to $v_{n+1} \in D$;

(c) moreover, if

$$
0<\rho<\frac{2 \mu \theta_{1}(\beta-\gamma r)\left(1-\theta_{2}\right)}{\theta_{2}^{2}(2 l s \delta+\gamma r)^{2}-4 \theta_{1}^{2} \beta(\beta-\gamma r)},
$$

then the sequences $\left\{u_{n}\right\}_{n \in \omega},\left\{v_{n}\right\}_{n \in \omega}$, and $\left\{w_{n}\right\}_{n \in \omega}$ defined by Algorithm 4.1 all strongly converge to $u$.

Proof (a) Note that (d2) implies that $\eta(u, u)=0$ and $\eta(u, v)=-\eta(v, u)$ for all $u, v \in D$. As in the proof of Theorem 3.1, by (3.9) and the Lipschitz continuity of $E$, we conclude that

$$
\|h x-h y\| \leq \frac{\alpha \delta}{\beta-\gamma r}\|E x-E y\| \leq \frac{\alpha \delta l}{\beta-\gamma r}\|x-y\|, \quad \forall x, y \in D,
$$

which together with (5.1) means that $h$ is a contraction mapping on $D$ and hence it has a unique fixed point $u \in D$ solving problem (2.2). We show the uniqueness of the solution 
for problem (2.2). Suppose that $\hat{u} \in D \backslash\{u\}$ is also a solution of problem (2.2). It follows that

$$
\langle N(E \hat{u}, F \hat{u}), \eta(v, \hat{u})\rangle+b(g \hat{u}, v)-b(g \hat{u}, \hat{u}) \geq 0, \quad \forall v \in D .
$$

Taking $v=\hat{u}$ in (2.2), $v=u$ in (5.3) and adding them together, by combining (b2), (b3), (c2), (c3), (d2), and (e1), we arrive at

$$
\begin{aligned}
0 \leq & \langle N(E \hat{u}, F \hat{u})-N(E u, F \hat{u}), \eta(u, \hat{u})\rangle+\langle N(E u, F \hat{u})-N(E u, F u), \eta(u, \hat{u})\rangle \\
& +b(g u-g \hat{u}, \hat{u})-b(g u-g \hat{u}, u) \\
\leq & (\alpha \delta l-\beta+\gamma r)\|u-\hat{u}\|^{2},
\end{aligned}
$$

which means that $\alpha \delta l-\beta+\gamma r \geq 0$, contradicting (5.1). Consequently, $u$ is the unique solution of problem (2.2).

(b) Similarly as in the proof of Theorem 4.1, it is easy to get that (b) holds.

(c) Let $u$ be the solution of problem (2.2). Define $C: D \rightarrow \mathbb{R} \cup\{+\infty\}$ by

$$
C(w)=K(u)-K(w)-\left\langle K^{\prime}(w), \eta(u, w)\right\rangle, \quad \forall w \in D .
$$

Since $K^{\prime}$ is $\eta$-strongly convex, we infer that

$$
C(w) \geq \frac{\mu}{2}\|w-u\|^{2}, \quad \forall w \in D .
$$

Setting $v=u$ in (4.3), we get that

$$
\begin{aligned}
& C\left(u_{n}\right)-C\left(w_{n}\right) \\
&= K\left(w_{n}\right)-K\left(u_{n}\right)-\left\langle K^{\prime}\left(u_{n}\right), \eta\left(w_{n}, u_{n}\right)\right\rangle+\left\langle K^{\prime}\left(w_{n}\right)-K^{\prime}\left(u_{n}\right), \eta\left(u, w_{n}\right)\right\rangle \\
& \geq \frac{\mu}{2}\left\|u_{n}-w_{n}\right\|^{2}+\frac{\alpha_{n} \rho}{1-\alpha_{n}}\left[\left\langle N\left(E v_{n}, F w_{n}\right), \eta\left(w_{n}, u\right)\right\rangle+b\left(g u_{n}, w_{n}\right)-b\left(g u_{n}, u\right)\right] \\
&= \frac{\mu}{2}\left\|u_{n}-w_{n}\right\|^{2}+\frac{\alpha_{n} \rho}{1-\alpha_{n}}\left[\left\langle N\left(E v_{n}, F w_{n}\right), \eta\left(w_{n}, u_{n}\right)\right\rangle\right. \\
&\left.+\left\langle N\left(E v_{n}, F w_{n}\right), \eta\left(u_{n}, u\right)\right\rangle+b\left(g u_{n}, w_{n}\right)-b\left(g u_{n}, u\right)\right] \\
&= \frac{\mu}{2}\left\|u_{n}-w_{n}\right\|^{2}+\frac{\alpha_{n} \rho}{1-\alpha_{n}}\left[\left\langle N\left(E v_{n}, F w_{n}\right)-N\left(E v_{n}, F u_{n}\right), \eta\left(w_{n}, u_{n}\right)\right\rangle\right. \\
&+\left\langle N\left(E v_{n}, F u_{n}\right)-N\left(E v_{n}, F u\right), \eta\left(w_{n}, u_{n}\right)\right\rangle \\
&+\left\langle N\left(E v_{n}, F w_{n}\right)-N\left(E v_{n}, F u_{n}\right), \eta\left(u_{n}, u\right)\right\rangle \\
&+\left\langle N\left(E v_{n}, F u_{n}\right)-N\left(E v_{n}, F u\right), \eta\left(u_{n}, u\right)\right\rangle \\
&\left.+\left\langle N\left(E v_{n}, F u\right), \eta\left(w_{n}, u\right)\right\rangle+b\left(g u_{n}, w_{n}\right)-b\left(g u_{n}, u\right)\right] \\
& \geq \frac{\mu}{2}\left\|u_{n}-w_{n}\right\|^{2}+\frac{\alpha_{n} \rho}{1-\alpha_{n}}\left[\beta\left\|u_{n}-w_{n}\right\|^{2}-2 t s \delta\left\|u_{n}-u\right\|\left\|u_{n}-w_{n}\right\|+\beta\left\|u_{n}-u\right\|^{2}\right. \\
&\left.-b\left(g u-g u_{n}, w_{n}\right)+b\left(g u-g u_{n}, u_{n}\right)-b\left(g u-g u_{n}, u_{n}\right)+b\left(g u-g u_{n}, u\right)\right] \\
& \geq \frac{\mu}{2}\left\|u_{n}-w_{n}\right\|^{2}+\frac{\alpha_{n} \rho}{1-\alpha_{n}}\left[\beta\left\|u_{n}-w_{n}\right\|^{2}-2 t s \delta\left\|u_{n}-u\right\|\left\|u_{n}-w_{n}\right\|\right.
\end{aligned}
$$




$$
\begin{aligned}
& \left.+\beta\left\|u_{n}-u\right\|^{2}-\gamma r\left\|u_{n}-u\right\|\left\|u_{n}-w_{n}\right\|-\gamma r\left\|u_{n}-u\right\|^{2}\right] \\
= & \frac{1}{2}\left(\mu+\frac{2 \alpha_{n} \rho \beta}{1-\alpha_{n}}\right)\left\|u_{n}-w_{n}\right\|^{2}-\frac{\alpha_{n} \rho}{1-\alpha_{n}}(2 t s \delta+\gamma r)\left\|u_{n}-u\right\|\left\|u_{n}-w_{n}\right\| \\
& +\frac{\alpha_{n} \rho}{1-\alpha_{n}}(\beta-\gamma r)\left\|u_{n}-u\right\|^{2} \\
\geq & \frac{1}{2\left(1-\alpha_{n}\right)}\left[2 \alpha_{n} \rho(\beta-\gamma r)-\frac{\alpha_{n}^{2} \rho^{2}(2 t s \delta+\gamma r)^{2}}{\mu-\mu \alpha_{n}+2 \alpha_{n} \rho \beta}\right]\left\|u_{n}-u\right\|^{2} \\
\geq & \frac{1}{2\left(1-\theta_{1}\right)}\left[2 \theta_{1} \rho(\beta-\gamma r)-\frac{\theta_{2}^{2} \rho^{2}(2 t s \delta+\gamma r)^{2}}{\mu-\mu \alpha_{n}+2 \theta_{1} \rho \beta}\right]\left\|u_{n}-u\right\|^{2}
\end{aligned}
$$

for all $n \in \omega$, where $\theta_{1}$ and $\theta_{2}$ are defined in (4.6). By using a similar argument, we can easily prove that

$$
\begin{aligned}
& C\left(w_{n}\right)-C\left(v_{n+1}\right) \geq \frac{1}{2\left(1-\theta_{1}\right)}\left[2 \theta_{1} \rho(\beta-\gamma r)-\frac{\theta_{2}^{2} \rho^{2}(2 t s \delta+\gamma r)^{2}}{\mu-\mu \alpha_{n}+2 \theta_{1} \rho \beta}\right]\left\|w_{n}-u\right\|^{2}, \\
& C\left(v_{n+1}\right)-C\left(u_{n+1}\right) \geq \frac{1}{2\left(1-\theta_{1}\right)}\left[2 \theta_{1} \rho(\beta-\gamma r)-\frac{\theta_{2}^{2} \rho^{2}(2 t s \delta+\gamma r)^{2}}{\mu-\mu \alpha_{n}+2 \theta_{1} \rho \beta}\right]\left\|v_{n+1}-u\right\|^{2}
\end{aligned}
$$

for all $n \in \omega$. Thus we have

$$
\begin{aligned}
& C\left(u_{n}\right)-C\left(u_{n+1}\right) \\
& \geq \frac{1}{2\left(1-\theta_{1}\right)}\left[2 \theta_{1} \rho(\beta-\gamma r)-\frac{\theta_{2}^{2} \rho^{2}(2 t s \delta+\gamma r)^{2}}{\mu-\mu \alpha_{n}+2 \theta_{1} \rho \beta}\right] \\
& \quad \times\left(\left\|u_{n}-u\right\|^{2}+\left\|w_{n}-u\right\|^{2}+\left\|v_{n+1}-u\right\|^{2}\right)
\end{aligned}
$$

for each $n \in \omega$. It follows from (5.2) that

$$
\frac{1}{2\left(1-\theta_{1}\right)}\left[2 \theta_{1} \rho(\beta-\gamma r)-\frac{\theta_{2}^{2} \rho^{2}(2 t s \delta+\gamma r)^{2}}{\mu-\mu \alpha_{n}+2 \theta_{1} \rho \beta}\right]>0
$$

Hence (5.5) and (5.6) yield that the sequence $\left\{C\left(u_{n}\right)\right\}_{n \in \omega}$ is nonincreasing, (5.4) ensures it is nonnegative, thus it converges to some number. Letting $n \rightarrow \infty$ in (5.5), we conclude that $\lim _{n \rightarrow \infty}\left\|u_{n}-u\right\|=\lim _{n \rightarrow \infty}\left\|w_{n}-u\right\|=\lim _{n \rightarrow \infty}\left\|v_{n+1}-u\right\|=0$. That is, the sequences $\left\{u_{n}\right\}_{n \in \omega},\left\{w_{n}\right\}_{n \in \omega}$ and $\left\{v_{n}\right\}_{n \in \omega}$ strongly converge to $u$ as $n \rightarrow \infty$. This completes the proof.

Acknowledgements

We are greatly indebted to the anonymous referee for helpful comments and stimulating hints.

\section{Funding}

This work was supported by the National Natural Science Foundation of China (No. 41701616).

Availability of data and materials

Not applicable.

Competing interests

The authors declare that they have no competing interests. 


\section{Author details}

'School of Mathematics, Dongbei University of Finance and Economics, Dalian, Liaoning 116025, People's Republic of China. ${ }^{2}$ Department of General Studies, Dalian University of Finance and Economics, Dalian, Liaoning 116000, People's Republic of China. ${ }^{3}$ Department of Mathematics, Tonghua Teachers College, Tonghua, Jilin 134002, People's Republic of China. ${ }^{4}$ Center for Studies of Marine Economy and Sustainable Development, Liaoning Normal University, Dalian, Liaoning 116029, People's Republic of China.

\section{Publisher's Note}

Springer Nature remains neutral with regard to jurisdictional claims in published maps and institutional affiliations.

Received: 22 July 2020 Accepted: 16 September 2020 Published online: 23 September 2020

\section{References}

1. Ansari, Q.H., Yao, J.C.: Iterative schemes for solving mixed variational-like inequalities. J. Optim. Theory Appl. 108, 527-541 (2001)

2. Aussel, D., Van, K.C., Salas, D.: Quasi-variational inequality problems over product sets with quasi-monotone operators SIAM J. Optim. 29, 1558-1577 (2019)

3. Ceng, L.C., Petrusel, A., Yao, J.C., Yao, Y.: Hybrid viscosity extragradient method for systems of variational inequalities, fixed points of nonexpansive mappings, zero points of accretive operators in Banach spaces. Fixed Point Theory 19, 487-502 (2018)

4. Ceng, L.C., Petrusel, A., Yao, J.C., Yao, Y.: Systems of variational inequalities with hierarchical variational inequality constraints for Lipschitzian pseudocontractions. Fixed Point Theory 20, 113-133 (2019)

5. Chang, S.S., Xiang, S.W.: On the existence of solutions for a class of quasi-bilinear variational inequalities. J. Syst. Sci. Math. Sci. 16, 136-140 (1996) (in Chinese)

6. Cohen, G.: Auxiliary problem principle extend to variational inequalities. J. Optim. Theory Appl. 59, 325-333 (1988)

7. Ding, X.P.: Algorithm of solutions for mixed nonlinear variational-like inequalities in reflexive Banach spaces. Appl. Math. Mech. 19, 521-529 (1998)

8. Ding, X.P.: General algorithm for nonlinear variational-like inequalities in reflexive Banach spaces. Indian J. Pure Appl. Math. 29, 109-120 (1998)

9. Ding, X.P., Tan, K.K.: A minimax inequality with applications to existence of equilibrium point and fixed point theorem. Colloq. Math. 63, 233-247 (1992)

10. Fan, K.: A generalization of Tychonoff's fixed point theorem. Math. Ann. 142, 305-310 (1961)

11. Hieu, D.V., Quy, P.K.: An inertial modified algorithm for solving variational inequalities. RAIRO Oper. Res. 54, 163-178 (2020)

12. Liu, Z., Chen, Z.S., Kang, S.M., Ume, J.S.: Existence and iterative approximations of solutions for mixed quasi-variational-like inequalities in Banach spaces. Nonlinear Anal. 69, 3259-3272 (2008)

13. Liu, Z., Ume, J.S., Kang, S.M.: Generalized nonlinear variational-like inequalities in reflexive Banach spaces. J. Optim. Theory Appl. 126, 157-174 (2005)

14. Shehu, Y., lyiola, O.S., Akaligwo, E.: Modified inertial methods for finding common solutions to variational inequality problems. Fixed Point Theory 20,683-702 (2019)

15. Thakur, B.S., Postolache, M.: Existence and approximation of solutions for generalized extended nonlinear variational inequalities. J. Inequal. Appl. 2013, Art. No. 590 (2013)

16. Yao, J.C.: Existence of generalized variational inequalities. Oper. Res. Lett. 15, 35-40 (1994)

17. Yao, Y., Postolache, M.: Iterative methods for pseudomonotone variational inequalities and fixed point problems. J. Optim. Theory Appl. 155, 273-287 (2012)

18. Yao, Y., Postolache, M., Liou, Y.C.: Variant extragradient-type method for monotone variational inequalities. Fixed Point Theory Appl. 2013, Art. No. 185 (2013)

19. Yao, Y., Postolache, M., Liou, Y.C., Yao, Z.: Construction algorithms for a class of monotone variational inequalities. Optim. Lett. 10, 1519-1528 (2016)

20. Yao, Y., Postolache, M., Yao, J.C.: An iterative algorithm for solving generalized variational inequalities and fixed points problems. Mathematics 7, Art. No. 61 (2019)

21. Yao, Y., Postolache, M., Yao, J.C.: Iterative algorithms for generalized variational inequalities. UPB Sci. Bull., Ser. A 81 3-16 (2019)

22. Yao, Y., Postolache, M., Yao, J.C.: Iterative algorithms for pseudomonotone variational inequalities and fixed point problems of pseudocontractive operators. Mathematics 7, Article ID 1189 (2019)

23. Yao, Y., Postolache, M., Yao, J.C.: Strong convergence of an extragradient algorithm for variational inequality and fixed point problems. UPB Sci. Bull., Ser. A 82, 3-12 (2020)

24. Zeng, L.C.: Iterative algorithm for finding approximate solutions of a class of mixed variational-like inequalities. Acta Math. Appl. Sin. 20, 477-486 (2004)

25. Zhang, L.L., Liu, Z., Kang, S.M.: On solvability of generalized nonlinear variational-like inequalities. J. Korean Math. Soc. 45, 163-176 (2008)

26. Zhao, X., Yao, J.C., Yao, Y.: A proximal algorithm for solving split monotone variational inclusions. UPB Sci. Bull., Ser. A $82,43-52(2020)$ 